\title{
Neurofilament light interaction with GluN1 modulates neurotransmission and schizophrenia-associated behaviors
}

\author{
Aidong Yuan 1,2, Veeranna 1,2, Henry Sershen 2,3, Balapal S. Basavarajappa ${ }^{2,4,6}$, John F. Smiley ${ }^{3}$, Audrey Hashim³,

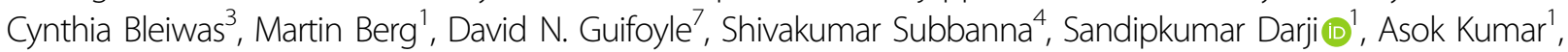 \\ Mala V. Rao ${ }^{1,2}$, Donald A. Wilson ${ }^{8,9,10}$, Jean-Pierre Julien ${ }^{11}$, Daniel C. Javitt ${ }^{5,12}$ and Ralph A. Nixon 1,2,10,13
}

\begin{abstract}
Neurofilament (NFL) proteins have recently been found to play unique roles in synapses. NFL is known to interact with the GluN1 subunit of N-methyl-D-aspartic acid (NMDAR) and be reduced in schizophrenia though functional consequences are unknown. Here we investigated whether the interaction of NFL with GluN1 modulates synaptic transmission and schizophrenia-associated behaviors. The interaction of NFL with GluN1 was assessed by means of molecular, pharmacological, electrophysiological, magnetic resonance spectroscopy (MRS), and schizophreniaassociated behavior analyses. NFL deficits cause an NMDAR hypofunction phenotype including abnormal hippocampal function, as seen in schizophrenia. NFL - / - deletion in mice reduces dendritic spines and GluN1 protein levels, elevates ubiquitin-dependent turnover of GluN1 and hippocampal glutamate measured by MRS, and depresses hippocampal long-term potentiation. NMDAR-related behaviors are also impaired, including pup retrieval, spatial and social memory, prepulse inhibition, night-time activity, and response to NMDAR antagonist, whereas motor deficits are minimal. Importantly, partially lowering NFL in NFL+/- mice to levels seen regionally in schizophrenia, induced similar but milder NMDAR-related synaptic and behavioral deficits. Our findings support an emerging view that central nervous system neurofilament subunits including NFL in the present report, serve distinctive, critical roles in synapses relevant to neuropsychiatric diseases.
\end{abstract}

\section{Introduction}

Neurofilaments (NF), unlike intermediate filaments of non-neuronal cells, are composed of four distinct subunits under complex regulation by phosphorylation ${ }^{1}$. The purpose of this added complexity of neuronal intermediate filaments has, until recently, been a puzzle. As linear heteropolymers, neurofilaments support the radial expansion of large myelinated axons. Because rare mutations of NFL (neurofilament light subunit, NEFL)

Correspondence: Aidong Yuan (yuan@nki.rfmh.org) or

Ralph A. Nixon (nixon@nki.rfmh.org)

${ }^{1}$ Center for Dementia Research, Nathan Kline Institute, Orangeburg, NY 10962, USA

${ }^{2}$ Departments of Psychiatry, New York University School of Medicine, New York, NY 10016, USA

Full list of author information is available at the end of the article. cause a subtype of the peripheral axonopathy, CharcotMarie-Tooth disease ${ }^{2-4}$, attention has previously focused mainly on roles of NF proteins in maintaining the elaborate axonal NF lattice of large caliber peripheral axons. In the central nervous system (CNS), however, NFs play a minor role in axon caliber expansion ${ }^{5,6}$ and recent evidence has shown that NF proteins form distinct protofilamentous assemblies within synaptic spines where the individual NF subunits differentially interact with specific neurotransmitter receptors and modulate their activity ${ }^{7}$.

Dendritic spines are believed to be an anatomical substrate for memory storage and synaptic transmission ${ }^{8,9}$ and a key feature of these spines is the postsynaptic density (PSD), which is critical for receptor stability and activity. The PSD is anchored and its functions are

\section{(c) The Author(s) 2018}

(c) (i) Open Access This article is licensed under a Creative Commons Attribution 4.0 International License, which permits use, sharing, adaptation, distribution and reproduction c. in any medium or format, as long as you give appropriate credit to the original author(s) and the source, provide a link to the Creative Commons license, and indicate if changes were made. The images or other third party material in this article are included in the article's Creative Commons license, unless indicated otherwise in a credit line to the material. If material is not included in the article's Creative Commons license and your intended use is not permitted by statutory regulation or exceeds the permitted use, you will need to obtain permission directly from the copyright holder. To view a copy of this license, visit http://creativecommons.org/licenses/by/4.0/. 
regulated by an actin-based cytoskeletal scaffold ${ }^{10}$ composed of proteins that interact directly with receptors, such as $N$-methyl-D-aspartic acid (NMDAR) ${ }^{11}$. Notably, mutations or deletions of many of the genes encoding proteins comprising the synaptic scaffold are believed to cause neuropsychiatric disorders, including schizophrenia, autism, and mental retardation (Supplementary Table S1). NF proteins have rarely been considered in relation to the synaptic scaffold, however, the evidence for a significant synaptic location of NF proteins and a wealth of recent proteomic data led us to propose that NFP is a core synaptic scaffold component ${ }^{6,7}$ and that NFL, which is known to interact with the GluN1 subunit of $\mathrm{NMDAR}^{12}$, may be essential for the proper functioning of this receptor. Supporting this possibility, an NFL interactome constructed from published data (Supplementary Figure S1) reveals direct interactions of NFL with many of the known postsynaptic scaffold proteins. Notably, a high proportion of these proteins are known to be altered in schizophrenia and other neuropsychiatric diseases (Supplementary Table S1).

The NMDAR, composed of four subunits including the essential GluN1 subunit ${ }^{13,14}$, is located on the postsynaptic membrane where it contributes to the expression of long-term potentiation (LTP) and synaptic plasticity thought to underlie learning and memory ${ }^{15}$. NMDAR hypofunction is believed to be involved in the pathophysiology of schizophrenia and genetic, biochemical, and pharmacological evidence documents reduced expression of particular subunits of the receptor, including GluN1, in the brains of individuals with schizophrenia ${ }^{16-21}$. Mice expressing reduced levels of the GluN1 subunit have been proposed as an animal model of schizophrenia ${ }^{22}$. NMDAR is also a major component of the PSD complex and its GluN1 subunit interacts with NFL in vitro and in non-neuronal cells transfected with NFL $\operatorname{protein}^{12}$. Moreover, like the GluN1 deficit in schizophrenia, substantially reduced levels of NFL are a remarkably consistent finding in affected regions of schizophrenic brain (Supplementary Table S2) although the functional significance of these deficits is unclear. Also, although we previously showed that the NFL subunit is an integral component of synapses, its functions at this site have not been previously addressed.

In the present report, we establish novel synaptic roles for the NFL subunit in maintaining spine morphology, stabilizing GluN1 levels, and modulating NMDAR function and related behavior. NFL gene deletion in mice lowered numbers and lengths of dendritic spines, depressed hippocampal long-term potentiation induction, and selectively depressed NMDAR-GluN1 protein levels and NMDAR-related behaviors while adaptively raising hippocampal glutamate levels. Importantly, we observed a similar range of NMDAR-related synaptic and behavioral deficits, albeit milder than in NFL-null mice, in NFL+/mice, in which brain NFL levels were lowered $40-50 \%$, a reduction within a range of NFL deficits seen in brain regions implicated in schizophrenia ${ }^{23}$ (Supplementary Table S2). Our findings reinforce an emerging concept that CNS NFL serve distinctive roles in synaptic transmission and behaviors related to the modulation of specific neurotransmitter receptors and we identify specific roles of the NFL subunit in NMDAR function.

\section{Materials and methods}

Generation of mutant animals, drugs, and antibodies

Please see Supplementary Information.

\section{Analytical methods}

Our published methods were used for all the procedures. Please see Supplementary Information.

\section{Results}

\section{NFL is essential for the maintenance of dendritic spine} structure and function

Immuno-electron microscopy (EM) studies confirmed ultrastructural colocalization of NFL and NFH on the same filament within dendrites (Fig. 1a, b) and postsynaptic terminals (Fig. 1c, d). Densitometric quantification of immunostained proteins revealed that levels of NFL proteins in hippocampal homogenates and synaptosomes from NFL-/- mice were undetectable, as expected (Fig. 1e). Levels of NFH were reduced to $22 \%$ of normal wild-type levels. By contrast, synaptosomes in these mice retained $\sim 50 \%$ of normal levels of NFM and $80 \%$ of normal INA, reflecting their known close physical and functional partnership ${ }^{24}$. Subunit stoichiometry in NFL-/ - hippocampus is comparable to previous analyses of optic axons ${ }^{25}$. An earlier study reporting NFM and NFH in NFL $-/-$ mice to be only $5 \%$ of WT levels ${ }^{26}$ can be explained by the fact that only phosphorylationdependent isoforms were used in this study, whereas we used phosphorylation-independent anti-NFM and antiNFH antibodies, which detect the larger total populations of each subunit.

We next used a rapid Golgi impregnation method to examine the effects of NFL deletion on the dendritic spines of hippocampal CA1 neurons (Fig. 1f, g). Spine density on dendritic arbors of CA1 neurons were significantly reduced in NFL $-/-$ mice compared with NFL $+/+$ mice $(p<0.0001, n=8)$ (Fig. 1h). Spine length was also significantly decreased $(p<0.001)$ (Fig. 1i). Similar changes in spine density and spine length were seen in dentate gyrus granule cells (Fig. $1 j-1$ ). In addition, path lengths of dendrites were significantly reduced in dentate gyrus granule cells of NFL-/- mice (Supplementary Table S3). NFL deletion had a greater effect on spines (lowered $41 \%$ in spine density and $24 \%$ in spine length) 


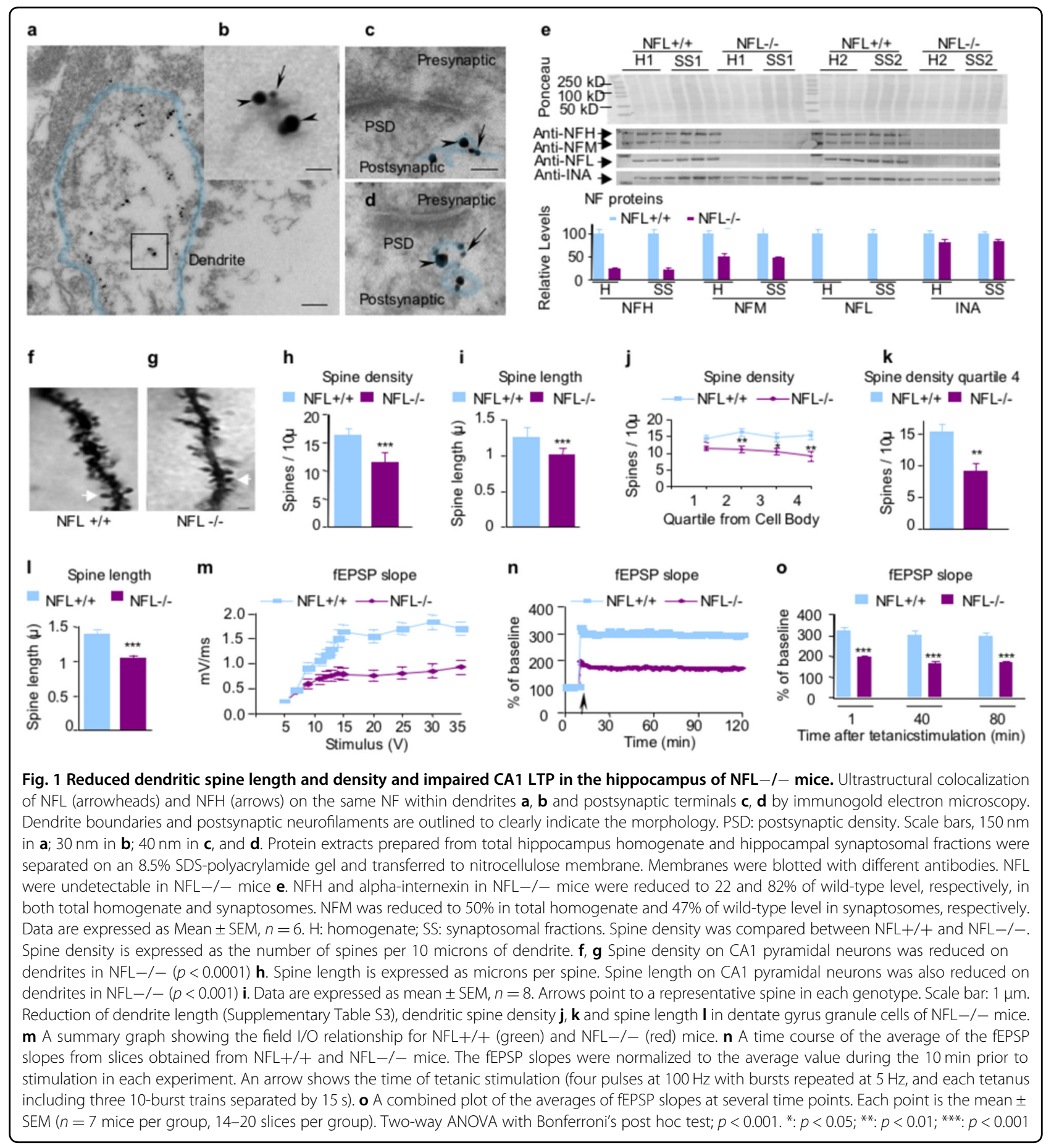

(Fig. 1k, l) than on dendrites (lowered 13\% in average path) (Supplementary Table S3), further suggesting the important structural role of NF proteins specifically within synaptic spines. Given the changes in spine morphology, we investigated whether or not NFL-/- mice have LTP deficits. Hippocampal basal synaptic neurotransmission and LTP in the Schaffer collateral pathway of hippocampal slices were found significantly impaired in
NFL $-/-$ as compared with NFL $+/+$ mice $(p<0.001, n=$ 7) (Fig. $1 \mathrm{~m}-\mathrm{o}$ ).

\section{NFL is essential for maintenance of NMDAR stability and activity at synapses}

Immuno-EM studies with anti-GluN1 antibody revealed reduced GluN1 immunoreactivity in hippocampal synapses (Fig. 2a-c). $\left[{ }^{3} \mathrm{H}\right] \mathrm{MDL} 105,519$ ligand binding to 


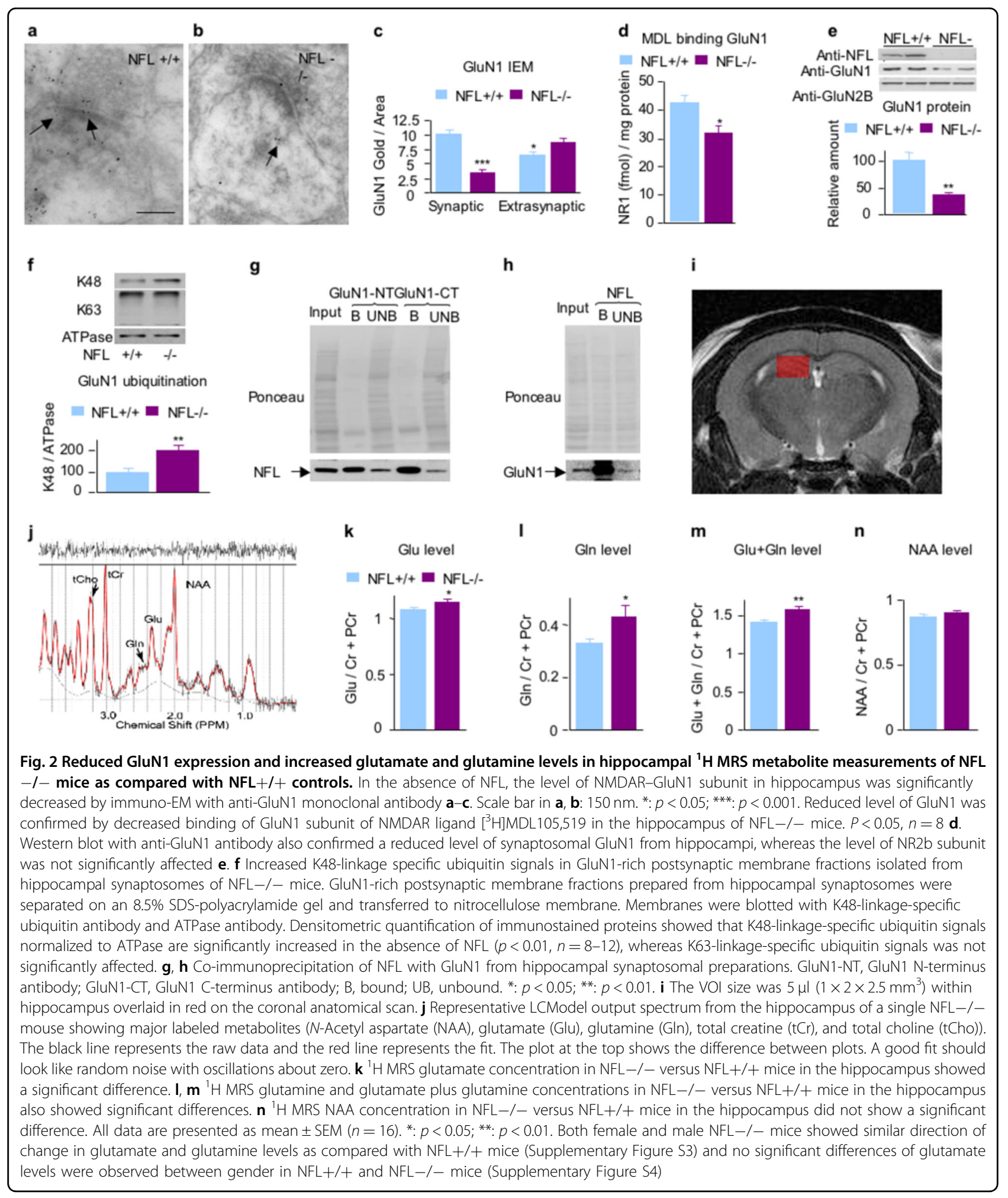

GluN1 was also significantly reduced in the hippocampal homogenates of NFL-/- as compared with NFL $+/+$ mice (Fig. 2d). Western blot studies showed that GluN1 subunit levels in hippocampal synaptosomes from
NFL $-/$ - mice were also substantially reduced (lowered $65 \%, p<0.001$ ) (Fig. 2e). Similar results were also observed in triple knockout mice lacking alpha-internexin, NFH, and NFL mice (Supplementary Figure S2). 
Densitometric quantification of immunostained proteins revealed that proteasome-degradation-related K48linkage specific ubiquitin signals normalized to ATPase signal, a marker of plasma membrane ${ }^{27}$, are significantly increased in the absence of NFL $(p<0.05$, mean \pm SEM, $n$ $=8-12$ ) (Fig. 2f), whereas non-proteasome-degradationrelated k63-linkage specific ubiquitin signals were not significantly altered ${ }^{28}$. In synaptosomal fractions, we also showed co-immunoprecipitation of GluN1 with NFL (Fig. 2g) and NFL with GluN1 (Fig. 2h), further substantiating in vivo interaction between GluN1 and NFL.

\section{Increased glutamate in the hippocampus of NFL-/- measured by ${ }^{1} \mathrm{H}$ MRS}

To determine whether lack of NFL affects glutamate level in hippocampus, we used in vivo magnetic resonance spectroscopy to measure regional content of relevant amino acids and metabolites in brain. The levels of Glu ( $p$ $<0.05, n=16)$ and Gln $(p<0.01)$ concentrations were significantly increased in the hippocampal region of NFL $-/-$ as compared with NFL mice (Fig. 2i-m). Hippocampal $\mathrm{N}$-acetyl aspartate (NAA) levels in NFL-/- mice did not differ significantly from NFL $+/+$ controls (Fig. 2n).

\section{NFL deletion causes diverse behavioral deficits related to NMDAR hypofunction}

To rule out the influence of a possible motor deficit on NMDAR-related behavior, we first performed grip strength and amphetamine-stimulated motor activity with NFL $-/$ - mice, which showed no significant impairment of motor capability in NFL-/- mice as compared with $\mathrm{NFL}+/+$ controls (Fig. 3a, b). By contrast, NFL $-/-$ mice displayed markedly reduced night-time locomotor activity at $9 \mathrm{pm}$ as compared with NFL $+/+$ controls $(P<0.001)$ (Fig. 3c). To implicate NMDAR in this locomotor deficit, we administered phencyclidine (PCP), an NMDAR antagonist that induces motor stimulant effects ${ }^{29}$. The motor stimulant effect of PCP was markedly decreased in NFL $-/-$ mice $(p<0.0001)$ (Fig. 3d), supporting the NMDAR relationship to locomotor deficits. We also found that a $6 \mathrm{~dB}$ prepulse of sound induced significantly greater inhibition of the startle reflex in NFL $+/+$ mice than in NFL-/- animals (Fig. 3e), indicating that NFL deletion leads to a deficit in PPI.

Pup retrieval is a social interaction test and a deficit in pup retrieval in mice has been used as an indicator of abnormal social interaction ${ }^{30-32}$. A pup retrieval test with $\mathrm{NFL}-/$ - mice showed that 9 out of $10 \mathrm{NFL}+/+$ mice finished the pup retrieval task within $10 \mathrm{~min}$, whereas only four out of $10 \mathrm{NFL}-/$ - mice did (Fig. 3f). Statistical analyses established a significant deficit in this innate maternal behavior in NFL- $/-$ mice $\left(\chi^{2}\right.$-test, $n=10, p<$ 0.05). NFL $+/+$ control mice displayed normal social memory, as demonstrated by a marked habituation (decreased exploration) during the first four trials and a striking dishabituation (increased exploration) upon the presentation of a novel animal on the 5th trial (Fig. 3g, h). By contrast, NFL- - - mice showed no significant habituation during the four exposures to the stimulus mouse or dishabituation to the novel stimulus mouse indicating a marked deficit in social interaction.

Object-placement task is hippocampus-dependent spatial memory test and glutamatergic transmission abnormalities can cause behavioral deficit in objectplacement memory ${ }^{33,34}$. Object placement was assessed in NFL-/ - mice with one-trial object-place recognition task in which the mice were allowed to investigate two objects for $5 \mathrm{~min}$ during a training session. After $4 \mathrm{~h}$ delay, a 5-min test trial was administered in which the mice were allowed to investigate the same two objects, but with one of the objects moved to a novel location. As expected, the $\mathrm{NFL}+/+$ mice investigated the moved object significantly more frequently than the stationary object $(p<0.01)$ (Fig. $3 i)$. By contrast, NFL $-/-$ mice did not show a preference for the moved object, suggesting they have impaired spatial memory of the training configuration. NFL-/and NFL $+/+$ mice showed similar numbers of explorations for both objects (Fig. 3j). We also assessed hippocampus-dependent memory with an object recognition task. This test is identical to the object-placement test, except that a novel object is substituted in the same location for one of the training objects during the test trials. Again, NFL $+/+$ mice showed a significant preference for the novel object, whereas NFL- - - did not ( $p$ $<0.01$ ) (Fig. 3k). There was no significant difference between the number of explorations for both objects by NFL $-/-$ and NFL $+/+$ mice (Fig. 3l).

\section{Partial NFL depletion in NFL $+/-$ mice induces NMDAR- related deficits of synaptic plasticity and behavior}

To determine the effects of a less-severe loss of NFL as might be associated with disease, we investigated certain of the above studied parameters in NFL $+/$ - as compared with wild-type control mice. Immunoblot analyses of NF subunit protein levels revealed that NFP stoichiometry changed in different ways in hippocampal homogenate and synaptosomes from NFL $+/-$ mice (Fig. 4a). Subunit levels were altered more in synaptosomes than in homogenates. NFL and NFM were reduced to half of the WT level $(p<0.01)$ : NFH and alpha-internexin were less affected (63\% and $77 \%$ of wild-type, respectively) than NFL and NFM. Previous studies also showed that the level of NFL protein decreased by $40 \%$ in the brain of NFL $+/-$ mice $^{35}$ and the levels of NFH, NFM, and INA in the cortex vary with age in the absence of $\mathrm{NFL}^{36}$. This extent of NF subunit change in synapses was associated, however, with significant synaptic dysfunction. 


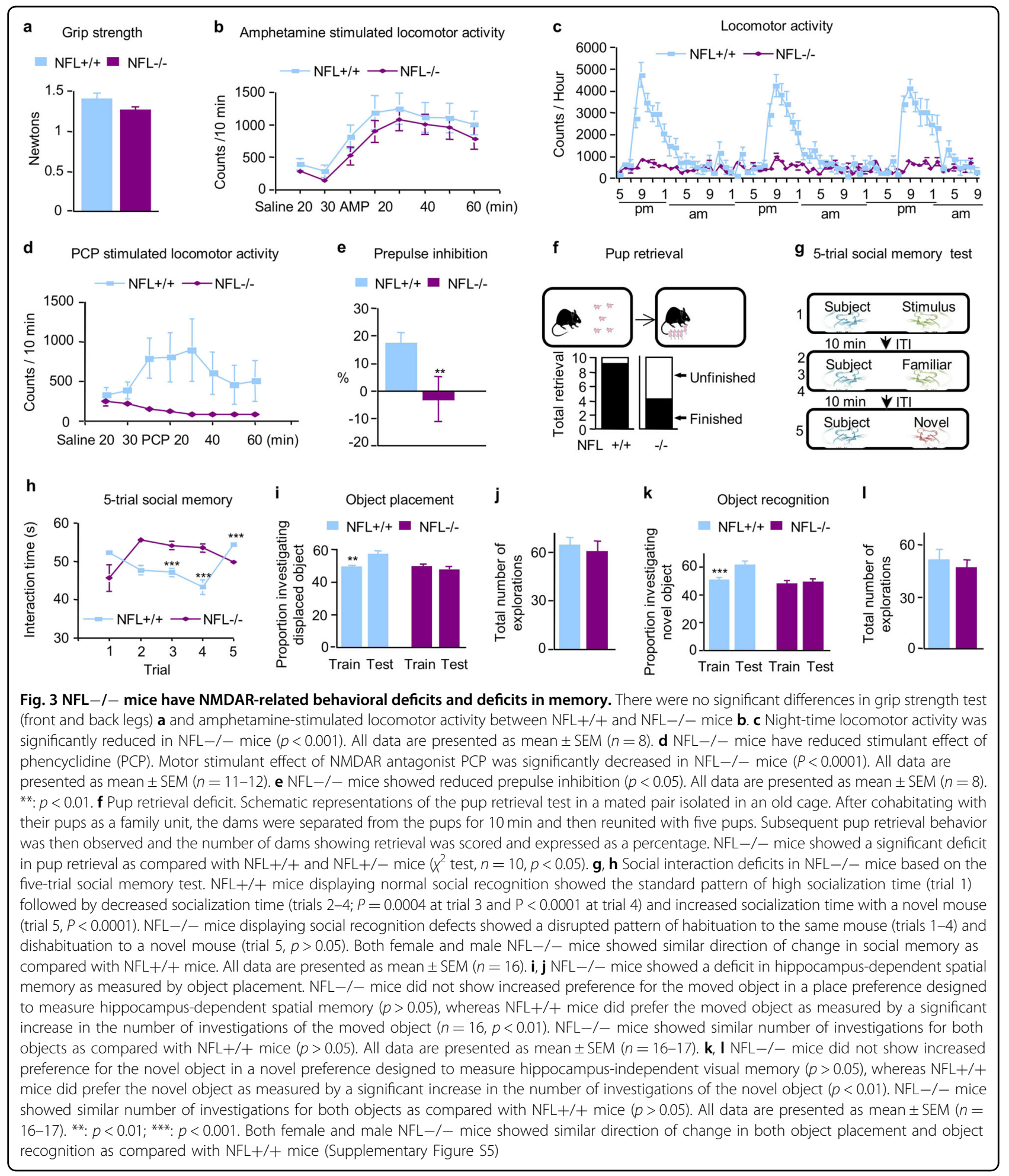

NFL $+/-$ mice exhibited significantly reduced spine density $(p<0.001, n=8)$ on dendritic arbors of CA1 neurons although spine lengths were unaltered (Fig. 4b). Similar effects of partial deletion of NFL were seen not only on spine density $(p<0.01)$ of dentate gyrus granule cells (Supplementary Figure S6a, b) but also with reduced spine length $(p<0.001)$ (Supplementary Figure S6c), indicating regional difference. Moreover, hippocampal basal synaptic neurotransmission and LTP in the Schaffer collateral pathway of hippocampal slices were significantly 


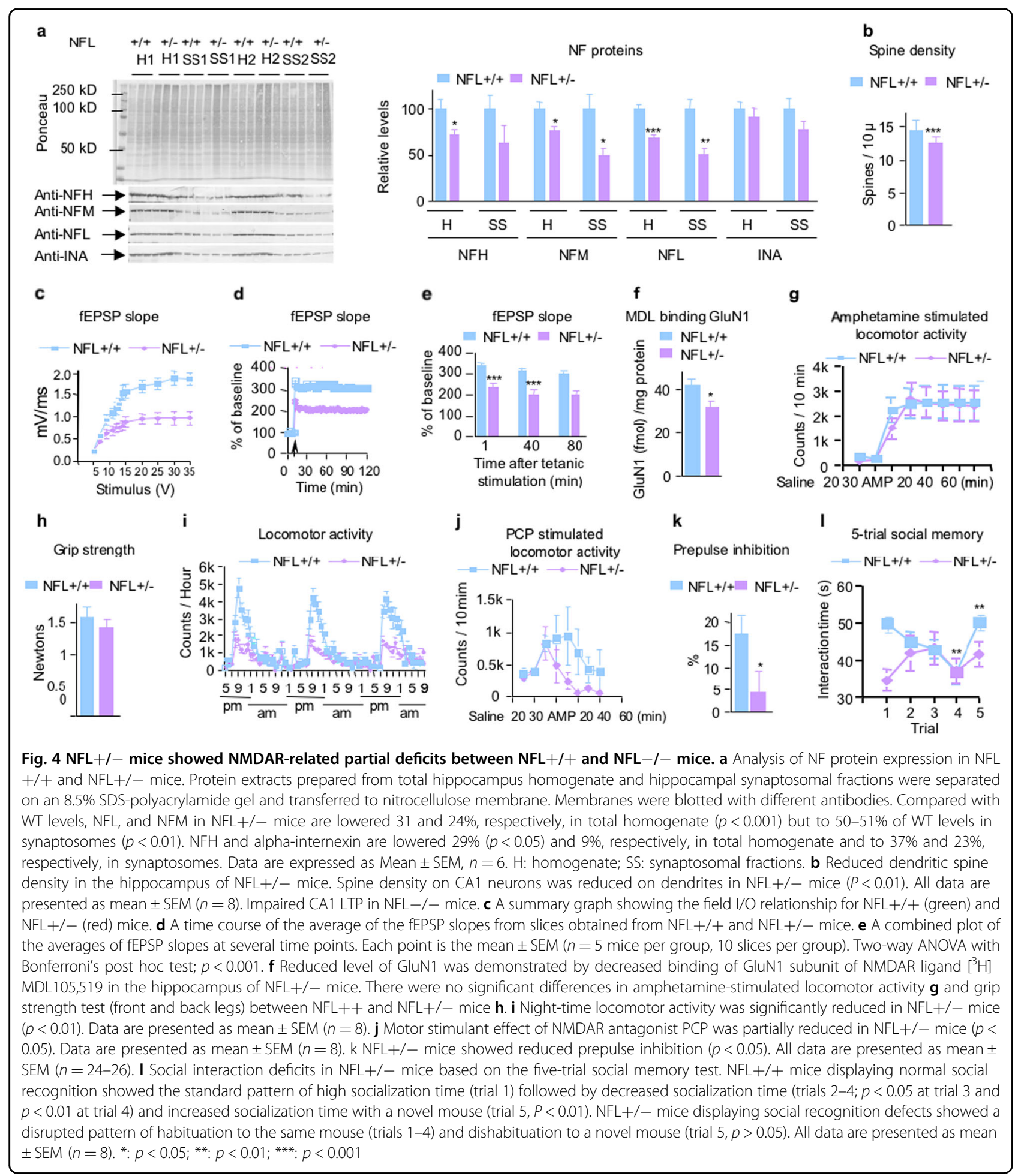

impaired in NFL $+/$ - as compared with NFL $+/+$ mice $(p$ $<0.001, n=5$ ) (Fig. 4c-e). GluN1 levels in the hippocampus of NFL $+/-$ mice determined by ligand-binding assay using $\left[{ }^{3} \mathrm{H}\right]$ MDL105,519 were significantly reduced (Fig. 4f). NFL $+/-$ mice showed a significantly diminished night-time locomotor activity at $9 \mathrm{pm}$ as compared with
NFL $+/+$ controls $(p<0.001, n=8)$ (Fig. 4i). The motor stimulant effect of PCP was also significantly decreased in NFL $+/-$ mice $(p<0.05, n=9)$ (Fig. 4j). We also found that a $3 \mathrm{~dB}$ prepulse of sound induced a significantly greater inhibition of the startle reflex in NFL $+/+$ mice than in NFL $+/-$ animals $(n=24-26, p<0.05)$ (Fig. $4 \mathrm{k})$, 
indicating that NFL partial deletion could lead to deficits in PPI. NFL $+/-$ mice also showed a significant deficit in social interaction $(p<0.05, n=8)$ (Fig. 4l). As shown in Fig. $4 \mathrm{~g}, \mathrm{~h}$, there was no significantly impaired motor capability in NFL $+/-$ mice as compared with NFL $+/+$ controls as measured by grip strength and amphetaminestimulated motor activity.

\section{Discussion}

In this report, we demonstrate that NFL is a critical component of synaptic spines and is essential for maintaining spine structural integrity and function. We present multiple lines of evidence showing that NFL modulates NMDAR level and function through a direct in vivo interaction with the GluN1 subunit, which protects against GluN1 ubiquitination and turnover. Finally, we show that even partial reductions in NFL levels, conceivably attainable in some neuropsychiatric states, have substantial synaptic and behavioral effects related to lowered GluN1 levels and diminished NMDAR activity.

NFL deletion is known to decrease axon caliber expansion and dendritic growth of large motoneurons during brain development ${ }^{26,37}$ and NFL's role in large myelinated peripheral axons has been a dominant focus of most neurofilament investigations. Consistent with these findings, we also found significantly reduced dendrite length, reduced spine density and spine length in dentate gyrus granule cells and reduced spine density and length in CA1 neurons of the hippocampus in NFL-/- mice even though neurofilaments in CNS neurons contribute minimally to axon caliber as demonstrated in our double and triple NF subunit deletion mice ${ }^{5,6}$. Dendritic spine pathology is known to be associated with various neuropsychiatric disorders that are also associated with NF protein alterations ${ }^{38-40}$ and our findings strongly suggest that loss of NFL from synapses may contribute to the spine instability and loss seen in some diseases. Notably, there is reduced dendritic spine density on cerebral cortical pyramidal neurons in schizophrenia ${ }^{41}$. In support, we saw that even a partial reduction in NFL (40\%), in the range of losses seen in vulnerable brain regions in schizophrenia, are associated with spine abnormalities.

We further showed that the spine alterations in NFLdeleted mice are accompanied by a marked impairment in basal synaptic transmission and LTP. For the first time, NFL-/- deletion in mice is shown to lower NMDAR-GluN1 protein levels and suppress NMDAR complex function while raising brain glutamate concentrations in hippocampus, presumably as a compensatory response ${ }^{42-45}$. Loss of NFL impaired NMDAR stability, synaptic signaling, and function in mediating behaviors known to be related to activity of this receptor. The selective decrease of GluN1 subunit associated with NFL deletion is consistent with previous evidence that exogenous NFL subunit co-expressed with GluN1 subunit in HEK 293 cells increased GluN1 cell surface expres$\operatorname{sion}^{12}$ and prevented its ubiquitination in vitro ${ }^{46}$. In our study, we demonstrated that K48-linkage-specific ubiquitin signals are significantly increased in GluN1-rich postsynaptic membranes from the hippocampi of NFL $-/-$ mice. This finding and the reduced levels of GluN1 suggest that, when NFL interaction with the receptor is lost, GluN1 degradation by the ubiquitinproteasome system is increased, consistent with evidence that GluN1 is a substrate of the UPS ${ }^{46,47}$.

Using proton magnetic resonance spectroscopy (MRS), a noninvasive method to measure neurotransmitter concentrations in vivo. we detected elevated glutamate and glutamine concentrations in the hippocampus, of NFL $-/-$ mice as compared with NFL $+/+$ controls. Although individual $1 \mathrm{H}-\mathrm{MRS}$ glutamate studies in schizophrenia have produced some inconsistent findings, a recent metaanalysis of proton MRS studies demonstrated schizophrenia is associated with elevations in glutamatergic metabolites across several brain regions ${ }^{44}$. Increased glutamate and glutamine concentrations in the hippocampus of NFL-/ - mice could be a result of decreased levels of NMDAR and could reflect the attempt of the system to compensate for postsynaptic glutamatergic hypofunction due to increased GABA disinhibition ${ }^{45}$.

NFL-/ - mice display a range of behavioral deficits associated with schizophrenia and in animal models of NMDAR hypofunction ${ }^{48}$ or GluN1 reduction ${ }^{49,50}$, including significant deficits of hippocampus-dependent social memory ${ }^{34}$. Pup retrieval behavior, a measure of hippocampus-dependent nonlearned innate behavior ${ }^{51,52}$ was markedly impaired. Also, object-placement and object-recognition tasks, which are hippocampusdependent spatial memory tests ${ }^{34,53}$ were deficient in NFL-/- mice. Prepulse inhibition, a characteristic feature of patients with schizophrenia and certain other psychiatric diseases, was also reduced in $\mathrm{NFL}-/-$ mice. NFL-/ - mice also have a reduction in night-time spontaneous locomotor activity that shows up most clearly at peak time points, suggesting that NFL has effects on locomotor systems that are not clearly a mimic of motor or sleep abnormalities found in schizophrenia ${ }^{54-56}$. Although early studies reported increased locomotor activity in mice expressing 5\% of normal levels of GluN1 ${ }^{22}$, recent investigation showed that locomotor activity in these mice is comparable to the locomotor activity of wild-type littermates in a familiar environment ${ }^{57}$. In fact, locomotor activity is not directly correlated to the levels of GluN1, as increased locomotor activity has been reported to be associated with both increase $^{58}$ or decrease ${ }^{59}$ of GluN1 levels. PCP is often used to model aspects of schizophrenia, inducing their prominent psychotomimetic effects by blocking 
neurotransmission at NMDAR-type glutamate receptors and inducing schizophrenia-related behaviors ${ }^{16,60-64}$. The present results showing a lack of response to PCPinduced locomotor activation in the NFL $-/-$ mice further suggest altered NMDAR-mediated mechanisms although one might expect increased sensitivity to PCPinduced locomotor effects in schizophrenic patients.

Our results generally parallel the deficits reported for GluN1 reduction models ${ }^{49,50}$. As NMDAR hypomorph mice show up to $95 \%$ reduced expression of GluN1 $1^{22,50}$, a reduction much greater than what has been shown in postmortem studies of schizophrenia brains ${ }^{65,66}$, heterozygous GluN1-/- mice exhibiting a 30\% reduced expression of GluN1 have been proposed to be more disease relevant in schizophrenia studies ${ }^{50}$. A $36 \%$ reduction of GluN1 protein was reported in postmortem brain (dorsolateral prefrontal cortex) from people with schizophrenia ${ }^{66}$. Although other studies reported inconsistent findings ${ }^{67,68}$, a recent meta-analysis of five studies of GluN1 protein, consisting of 95 subjects with schizophrenia and 95 controls, indicates a significant decrease in expression of GluN1 protein in schizophrenia subjects relative to controls ${ }^{68}$. Consistent with these findings from human studies, a 30\% reduction of GluN1 protein in rat hippocampus induced by GluN1-antisense treatment produced deficits of prepulse inhibition, a well-defined finding in schizophrenia ${ }^{49}$.

Modeling closely the NFL declines reported in affected brain regions in schizophrenia, NFL $+/$ - mice exhibiting a $40 \%$ reduction in NFL levels also have significant NMDAR-related deficits. Levels of NFL protein are decreased $\sim 50 \%$ (statistically significant) by western blot in the dorsolateral prefrontal cortex and $\sim 40 \%$ (not significant) in the anterior cingulate cortex from patients with schizophrenia ${ }^{23}$. Although $15 \%$ increase of NFL transcript was initially reported (these effects were across the collapsed layers and none showed isolated significant changes in a specific isodense band) ${ }^{23}$, a later study by the same group demonstrated $\sim 20 \%$ (statistically significant) reduction of NFL transcript in the schizophrenic dorsolateral prefrontal cortex (layer V) ${ }^{69}$. Many recent unbiased proteomic studies also demonstrated consistent and significant reduction of NFL subunits in brain regions essential for the cognitive and behavior functions affected in schizophrenia (Supplementary Table S2). The fact that NFL, NFM and NFH genes map to chromosomal regions (8p21, 8p22, and 22q12, respectively) that are strongly implicated in schizophrenia raises the possibility of an involvement of NF proteins in this disease $\mathrm{e}^{70,71}$. NFL showed consistently reduced expression in three brain regions (anterior cingulate gyrus, motor cortex, and

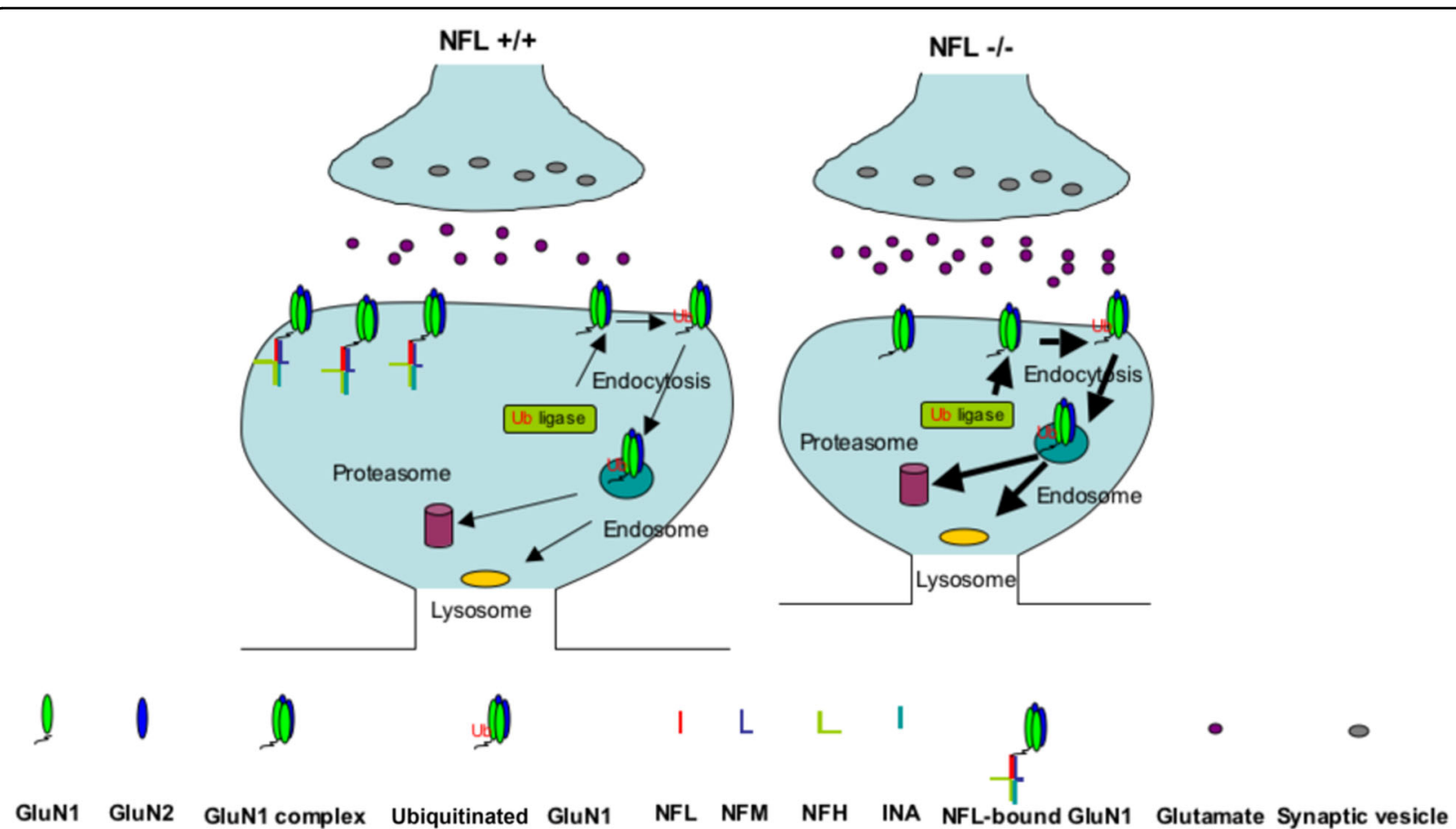

Fig. 5 Model of NMDAR binding to NFL. On the basis of collective findings on NF scaffolding functions and our GluN1 data on NFL deletion mice, we propose a model by which NFL acts in synaptic terminals to bind NMDAR on postsynaptic terminals to stabilize the level by protecting against ubiquitination of GluN1. In NFL+/+, GluN1 subunits are physiologically ubiquitinated and degraded as indicated by thin arrows; in NFL $-/-$, greater ubiquitination and degradation of GluN1 leading to reduced NMDAR function as indicated by thick arrows 
thalamus) following gene expression analysis of postmortem brain tissue of autism patients ${ }^{72}$. NFL also showed genetic association with autism in Caucation families ${ }^{72}$. Our findings are consistent with a model (Fig. 5) in which NFL within a synaptic cytoskeletal lattice binds to the GluN1 receptor and maintains its levels on the postsynaptic surface by preventing its ubiquitination and turnover. Lowered levels of NFL, a substrate for calpains $^{73}$, proteasome ${ }^{74}$, and autophagy ${ }^{75}$ (Rao et al. to be submitted), result in greater ubiquitination and degradation of GluN1 receptors and possibly disruption of key PP1-CamKII interactions with the NMDAR leading to hypofunction of NMDAR signaling. Recent studies of synaptic scaffold proteins suggest that they may form large protein networks and play a major role in synaptic function including the trafficking, anchoring, clustering, and stability of glutamate receptors ${ }^{76}$. Our present results together with previous findings ${ }^{77-79}$ document the likely interactions of NFL, and NF proteins in general, in the scaffold of networked proteins (Supplementary Figure S1) and in the dysfunction of the synaptic scaffold proteins as a molecular basis for severe neuropsychiatric disorders (Supplementary Table S1).

\section{Acknowledgements}

This work was supported by Grant 5R01AG005604 (R.A.N.) from the National Institutes on Aging. The MR system was supported in part by $\mathrm{NIH}$ grant 1S10RR023534-01. B.S.B. is supported by NIH grant (R01 AA019443).

\section{Author details}

${ }^{1}$ Center for Dementia Research, Nathan Kline Institute, Orangeburg, NY 10962 USA. ${ }^{2}$ Departments of Psychiatry, New York University School of Medicine, New York, NY 10016, USA. ${ }^{3}$ Neurochemistry Division, Nathan Kline Institute, Orangeburg, NY 10962, USA. ${ }^{4}$ Analytical Psychopharmacology Division, Nathan Kline Institute, Orangeburg, NY 10962, USA. ${ }^{5}$ Department of Psychiatry, College of Physicians \& Surgeons, Columbia University, New York, NY 10032, USA. ${ }^{6}$ New York State Psychiatric Institute, New York, NY 10032, USA. ${ }^{7}$ Center for Biomedical Imaging and Neuromodulation, Nathan Kline Institute, Orangeburg, NY 10962, USA. ${ }^{8}$ Emotional Brain Institute, Nathan Kline Institute, Orangeburg, NY 10962, USA. ${ }^{9}$ Child and Adolescent Psychiatry, New York University School of Medicine, New York, NY 10016, USA. ${ }^{10}$ Neuroscience Institute, New York University School of Medicine, New York, NY 10016, USA.

${ }^{11}$ Centre de Recherche du Centre Hospitalier de I'Université Laval, Département d'anatomie et physiologie de I'Université Laval, 2795 boul. Laurier, Québec G1V 4G2, Canada. ${ }^{12}$ Schizophrenia Research, Nathan Kline Institute, Orangeburg, NY 10962, USA. ${ }^{13}$ Department of Cell Biology, New York University School of Medicine, New York, NY 10016, USA

\section{Author Contributions}

A.Y., V., H.S., B.S.B., J.F.S., D.A.W., R.A.N. designed research; A.Y., V., H.S., B.S.B., J.F.S., A.H., C.B., S.D., A.K., and M.B. performed research; D.N.G. acquired and processed the MRS data; A.Y., V, H.S., B.S.B., J.F.S., A.H., M.V.R, J-P.J., D.C.J., and R.A. N. analyzed data; A.Y. and R.A.N. wrote the paper.

\section{Conflict of interest}

The authors declare that they have no conflict of interest.

\section{Publisher's note}

Springer Nature remains neutral with regard to jurisdictional claims in published maps and institutional affiliations.
Supplementary Information accompanies this paper at (https://doi.org/ 10.1038/s41398-018-0194-7)

Received: 23 May 2018 Accepted: 8 June 2018

Published online: 24 August 2018

\section{References}

1. Yuan A., Rao M. V., Veeranna, Nixon RA. Neurofilaments and neurofilament proteins in health and disease. Cold Spring Harb. Perspect. Biol. 2017; 9:pii: a018309.

2. Mersiyanova, I. V. et al. A new variant of Charcot-Marie-Tooth disease type 2 is probably the result of a mutation in the neurofilament-light gene. Am. J. Hum. Genet. 67, 37-46 (2000).

3. Abe, A. et al. Neurofilament light chain polypeptide gene mutations in Charcot-Marie-Tooth disease: nonsense mutation probably causes a recessive phenotype. J. Hum. Genet. 54, 94-97 (2009).

4. Yum, S. W., Zhang, J., Mo, K., Li, J. \& Scherer, S. S. A novel recessive Nefl mutation causes a severe, early-onset axonal neuropathy. Ann. Neurol. 66 759-770 (2009).

5. Dyakin, V. V. et al. The contributions of myelin and axonal caliber to transverse relaxation time in shiverer and neurofilament-deficient mouse models. Neuroimage 51, 1098-1105 (2010).

6. Yuan, A. \& Nixon, R. A. Specialized roles of neurofilament proteins in synapses: relevance to neuropsychiatric disorders. Brain Res. Bull. 126, 334-346 (2016).

7. Yuan, A. et al. Neurofilament subunits are integral components of synapses and modulate neurotransmission and behavior in vivo. Mol. Psychiatry 20, 986-994 (2015).

8. Engert, F. \& Bonhoeffer, T. Dendritic spine changes associated with hippocampal long-term synaptic plasticity. Nature 399, 66-70 (1999).

9. Huang, Z. H., Wu, H. J., Yeh, C. C., Chou, Y. C. \& Chang, Y. C. Dendritic spines of developing rat cortical neurons in culture. Chin. J. Physiol. 49, 39-45 (2006).

10. Qualmann, B., Boeckers, T. M., Jeromin, M., Gundelfinger, E. D. \& Kessels, M. M. Linkage of the actin cytoskeleton to the postsynaptic density via direct interactions of Abp1 with the ProSAP/Shank family. J. Neurosci. 24, 2481-2495 (2004).

11. Bassand, P., Bernard, A., Rafiki, A., Gayet, D. \& Khrestchatisky, M. Differential interaction of the $\mathrm{TSXV}$ motifs of the NR1 and NR2A NMDA receptor subunits with PSD-95 and SAP97. Eur. J. Neurosci. 11, 2031-2043 (1999).

12. Ehlers, M. D., Fung, E. T., O'Brien, R. J. \& Huganir, R. L. Splice variant-specific interaction of the NMDA receptor subunit NR1 with neuronal intermediate filaments. J. Neurosci. 18, 720-730 (1998),

13. Goebel, D. J. \& Poosch, M. S. NMDA receptor subunit gene expression in the rat brain: a quantitative analysis of endogenous mRNA levels of NR1Com, NR2A, NR2B, NR2C, NR2D and NR3A. Brain Res. Mol. Brain Res. 69, 164-170 (1999).

14. McBain, C. J. \& Mayer, M. L. N-methyl-D-aspartic acid receptor structure and function. Physiol. Rev. 74, 723-760 (1994).

15. Li, F. \& Tsien, J. Z. Memory and the NMDA receptors. N. Engl. J. Med. 361 302-303 (2009).

16. Javitt, D. C. \& Zukin, S. R. Recent advances in the phencyclidine model of schizophrenia. Am. J. Psychiatry 148, 1301-1308 (1991).

17. Law, A. J. \& Deakin, J. F. Asymmetrical reductions of hippocampal NMDAR1 glutamate receptor mRNA in the psychoses. Neuroreport 12, 2971-2974 (2001).

18. Pilowsky, L. S. et al. First in vivo evidence of an NMDA receptor deficit in medication-free schizophrenic patients. Mol. Psychiatry 11, 118-119 (2006).

19. Tarabeux, J. et al. Rare mutations in N-methyl-D-aspartate glutamate receptors in autism spectrum disorders and schizophrenia. Transl. Psychiatry 1, e55 (2011).

20. Fromer, M. et al. De novo mutations in schizophrenia implicate synaptic networks. Nature 506, 179-184 (2014).

21. Kantrowitz, J. T. et al. High dose D-serine in the treatment of schizophrenia. Schizophr. Res. 121, 125-130 (2010).

22. Mohn, A. R., Gainetdinov, R. R., Caron, M. G. \& Koller, B. H. Mice with reduced NMDA receptor expression display behaviors related to schizophrenia. Cell $\mathbf{9 8}$ 427-436 (1999)

23. Kristiansen, L. V., Beneyto, M., Haroutunian, V. \& Meador-Woodruff, J. H. Changes in NMDA receptor subunits and interacting PSD proteins in 
dorsolateral prefrontal and anterior cingulate cortex indicate abnormal regional expression in schizophrenia. Mol. Psychiatry 11, 737-747 (2006). 705.

24. Yuan, A. et al. Alpha-internexin is structurally and functionally associated with the neurofilament triplet proteins in the mature CNS. J. Neurosci. 26, 10006-10019 (2006)

25. Yuan, A., Rao, M. V., Kumar, A., Julien, J. P. \& Nixon, R. A. Neurofilament transport in vivo minimally requires hetero-oligomer formation. J. Neurosci. 23, 9452-9458 (2003).

26. Zhu, Q., Couillard-Despres, S. \& Julien, J. P. Delayed maturation of regenerating myelinated axons in mice lacking neurofilaments. Exp. Neurol. 148, 299-316 (1997).

27. Therien, A. G. \& Blostein, R. Mechanisms of sodium pump regulation. Am. J. Physiol. Cell Physiol. 279, C541-C566 (2000).

28. Spence, J., Sadis, S., Haas, A. L. \& Finley, D. A ubiquitin mutant with specific defects in DNA repair and multiubiquitination. Mol. Cell Biol. 15, 1265-1273 (1995).

29. Jackson, D. M., Johansson, C., Lindgren, L. M. \& Bengtsson, A. Dopamine receptor antagonists block amphetamine and phencyclidine-induced motor stimulation in rats. Pharmacol. Biochem. Behav. 48, 465-471 (1994).

30. Leckman, J. F. \& Herman, A. E. Maternal behavior and developmental psychopathology. Biol. Psychiatry 51, 27-43 (2002).

31. Girirajan, S. \& Elsea, S. H. Abnormal maternal behavior, altered sociability, and impaired serotonin metabolism in Rai1-transgenic mice. Mamm. Genome. 20, 247-255 (2009)

32. Trezza, V., Campolongo, P. \& Vanderschuren, L. J. Evaluating the rewarding nature of social interactions in laboratory animals. Dev. Cogn. Neurosci. 1, 444-458 (2011).

33. Assini, F. L., Duzzioni, M. \& Takahashi, R. N. Object location memory in mice: pharmacological validation and further evidence of hippocampal CA1 participation. Behav. Brain Res. 204, 206-211 (2009).

34. Kaur, G. et al. Glutamatergic transmission aberration: a major cause of behavioral deficits in a murine model of Down's syndrome. J. Neurosci. 34, 5099-5106 (2014).

35. Nguyen, M. D., Lariviere, R. C. \& Julien, J. P. Reduction of axonal caliber does not alleviate motor neuron disease caused by mutant superoxide dismutase 1. Proc. Natl. Acad. Sci. USA 97, 12306-12311 (2000).

36. Liu, Y. et al. Cytoskeletal changes during development and aging in the cortex of neurofilament light protein knockout mice. J. Comp. Neurol. 521, 1817-1827 (2013).

37. Zhang, Z., Casey, D. M., Julien, J. P. \& Xu, Z. Normal dendritic arborization in spinal motoneurons requires neurofilament subunit L. J. Comp. Neurol. 450 144-152 (2002)

38. Glantz, L. A. \& Lewis, D. A. Decreased dendritic spine density on prefrontal cortical pyramidal neurons in schizophrenia. Arch. Gen. Psychiatry 57, 65-73 (2000).

39. Perez-Cruz, C. et al. Reduced spine density in specific regions of CA1 pyramidal neurons in two transgenic mouse models of Alzheimer's disease. J. Neurosci. 31, 3926-3934 (2011).

40. Durand, C. M. et al. SHANK3 mutations identified in autism lead to modification of dendritic spine morphology via an actin-dependent mechanism. Mol. Psychiatry 17, 71-84 (2012).

41. Garey, L. J. et al. Reduced dendritic spine density on cerebral cortical pyramidal neurons in schizophrenia. J. Neurol. Neurosurg. Psychiatry 65, 446-453 (1998).

42. van Elst, L. T. et al. Increased prefrontal and hippocampal glutamate concentration in schizophrenia: evidence from a magnetic resonance spectroscopy study. Biol. Psychiatry 58, 724-730 (2005).

43. de la Fuente-Sandoval, C. et al. Higher levels of glutamate in the associativestriatum of subjects with prodromal symptoms of schizophrenia and patients with first-episode psychosis. Neuropsychopharmacology 36, 1781-1791 (2011)

44. Merritt, K., Egerton, A., Kempton, M. J., Taylor, M. J. \& McGuire, P. K. Nature of glutamate alterations in schizophrenia: a meta-analysis of proton magnetic resonance spectroscopy studies. JAMA Psychiatry 73, 665-674 (2016).

45. Homayoun, H. \& Moghaddam, B. NMDA receptor hypofunction produces opposite effects on prefrontal cortex interneurons and pyramidal neurons. J. Neurosci. 27, 11496-11500 (2007).

46. Ratnam, J. \& Teichberg, V. I. Neurofilament-light increases the cell surface expression of the $\mathrm{N}$-methyl-D-aspartate receptor and prevents its ubiquitination. J. Neurochem. 92, 878-885 (2005).

47. Kato, A., Rouach, N., Nicoll, R. A. \& Bredt, D. S. Activity-dependent NMDA receptor degradation mediated by retrotranslocation and ubiquitination. Proc Natl. Acad. Sci. USA 102, 5600-5605 (2005).
48. Balu, D. T. et al. Multiple risk pathways for schizophrenia converge in serine racemase knockout mice, a mouse model of NMDA receptor hypofunction. Proc. Natl. Acad. Sci. USA 110, E2400-E2409 (2013).

49. Inada, K. et al. Antisense hippocampal knockdown of NMDA-NR1 by HVJliposome vector induces deficit of prepulse inhibition but not of spatial memory. Neurosci. Res. 45, 473-481 (2003).

50. Featherstone, R. E. et al. Mice with subtle reduction of NMDA NR1 receptor subunit expression have a selective decrease in mismatch negativity: Implications for schizophrenia prodromal population. Neurobiol. Dis. 73, 289-295 (2015).

51. Kimble, D. P., Rogers, L. \& Hendrickson, C. W. Hippocampal lesions disrupt maternal, not sexual, behavior in the albino rat. J. Comp. Physiol. Psychol. 63 401-407 (1967).

52. Terlecki, L. J. \& Sainsbury, R. S. Effects of fimbria lesions on maternal behavior in the rat. Physiol. Behav. 21, 89-97 (1978).

53. Cohen, S. J. et al. The rodent hippocampus is essential for nonspatial object memory. Curr. Biol. 23, 1685-1690 (2013).

54. Cuesta, M. J. et al. Motor abnormalities in first-episode psychosis patients and long-term psychosocial functioning. Schizophr. Res. (2017). https://doi.org/ 10.1016/j.schres.2017.08.050

55. Kantrowitz, J. T. et al. The importance of a good night's sleep: an open-label trial of the sodium salt of gamma-hydroxybutyric acid in insomnia associated with schizophrenia. Schizophr. Res. 120, 225-226 (2010).

56. Cohrs, S. Sleep disturbances in patients with schizophrenia: impact and effect of antipsychotics. CNS Drugs 22, 939-962 (2008).

57. Halene, T. B. et al. Assessment of NMDA receptor NR1 subunit hypofunction in mice as a model for schizophrenia. Genes Brain Behav. 8, 661-675 (2009).

58. Hanania, T., Hillman, G. R. \& Johnson, K. M. Augmentation of locomotor activity by chronic phencyclidine is associated with an increase in striatal NMDA receptor function and an upregulation of the NR1 receptor subunit. Synapse 31, 229-239 (1999).

59. Yasuda, K. et al. Schizophrenia-like phenotypes in mice with NMDA receptor ablation in intralaminar thalamic nucleus cells and gene therapy-based reversal in adults. Transl. Psychiatry 7, e1047 (2017).

60. Javitt, D. C., Jotkowitz, A., Sircar, R. \& Zukin, S. R. Non-competitive regulation of phencyclidine/sigma-receptors by the $\mathrm{N}$-methyl-D-aspartate receptor antagonist D-(-)-2-amino-5-phosphonovaleric acid. Neurosci. Lett. 78, 193-198 (1987).

61. Olney, J. W. \& Farber, N. B. Glutamate receptor dysfunction and schizophrenia. Arch. Gen. Psychiatry 52, 998-1007 (1995).

62. Newcomer, J. W. et al. Ketamine-induced NMDA receptor hypofunction as a model of memory impairment and psychosis. Neuropsychopharmacology 20, 106-118 (1999).

63. Jentsch, J. D. \& Roth, R. H. The neuropsychopharmacology of phencyclidine: from NMDA receptor hypofunction to the dopamine hypothesis of schizophrenia. Neuropsychopharmacology 20, 201-225 (1999).

64. Bickel, S. \& Javitt, D. C. Neurophysiological and neurochemical animal models of schizophrenia: focus on glutamate. Behav. Brain Res. 204, 352-362 (2009).

65. Gao, X. M. et al. Ionotropic glutamate receptors and expression of N-methyl-Daspartate receptor subunits in subregions of human hippocampus: effects of schizophrenia. Am. J. Psychiatry 157, 1141-1149 (2000).

66. Weickert, C. S. et al. Molecular evidence of N-methyl-D-aspartate receptor hypofunction in schizophrenia. Mol. Psychiatry 18, 1185-1192 (2013).

67. Henson, M. A. et al. Developmental regulation of the NMDA receptor subunits, NR3A and NR1, in human prefrontal cortex. Cereb. Cortex 18, 2560-2573 (2008).

68. Catts, V. S., Lai, Y. L., Weickert, C. S., Weickert, T. W. \& Catts, S. V. A quantitative review of the postmortem evidence for decreased cortical N-methyl-Daspartate receptor expression levels in schizophrenia: how can we link molecular abnormalities to mismatch negativity deficits? Biol. Psychol. 116, 57-67 (2016).

69. Beneyto, M. \& Meador-Woodruff, J. H. Lamina-specific abnormalities of NMDA receptor-associated postsynaptic protein transcripts in the prefrontal cortex in schizophrenia and bipolar disorder. Neuropsychopharmacology 33, 2175-2186 (2008).

70. Badner, J. A. \& Gershon, E. S. Meta-analysis of whole-genome linkage scans of bipolar disorder and schizophrenia. Mol. Psychiatry 7, 405-411 (2002).

71. Lewis, C. M. et al. Genome scan meta-analysis of schizophrenia and bipolar disorder, part II: Schizophrenia. Am. J. Hum. Genet. 73, 34-48 (2003).

72. Anitha, A. et al. Brain region-specific altered expression and association of mitochondria-related genes in autism. Mol. Autism 3, 12 (2012). 
73. Kunz, S. et al. The calpain inhibitor MDL 28170 prevents inflammation-induced neurofilament light chain breakdown in the spinal cord and reduces thermal hyperalgesia. Pain 110, 409-418 (2004).

74. Balastik, M. et al. Deficiency in ubiquitin ligase TRIM2 causes accumulation of neurofilament light chain and neurodegeneration. Proc. Natl. Acad. Sci. USA 105, 12016-12021 (2008).

75. Chen, J. X. et al. Induction of autophagy by TOCP in differentiated human neuroblastoma cells lead to degradation of cytoskeletal components and inhibition of neurite outgrowth. Toxicology 310, 92-97 (2013).

76. Verpelli, C., Schmeisser, M. J., Sala, C. \& Boeckers, T. M. Scaffold proteins at the postsynaptic density. Adv. Exp. Med. Biol. 970, 29-61 (2012).
77. Husi, H., Ward, M. A., Choudhary, J. S., Blackstock, W. P. \& Grant, S. G. Proteomic analysis of NMDA receptor-adhesion protein signaling complexes. Nat. Neurosci. 3, 661-669 (2000).

78. Coumans J. V., Palanisamy S. K., McFarlane J., Moens P. D. Proteomic and microscopic strategies towards the analysis of the cytoskeletal networks in major neuropsychiatric disorders. Int. J. Mol. Sci. 2016; 17:pii: E581.

79. Alfieri, A. et al. Synaptic interactome mining reveals p140Cap as a new hub for PSD proteins involved in psychiatric and neurological disorders. Front. Mol. Neurosci. 10, 212 (2017) 